\title{
Numerical differentiation on irregular grids
}

\author{
Samer Riachy* Mamadou Mboup ${ }^{* *}$ Jean-Pierre Richard ${ }^{* * *}$ \\ * ECS-Lab EA 3649, ENSEA \& EPI Non-A, INRIA, 6 avenue \\ du Ponceau, 95014 Cergy-Pontoise, France.(Samer.Riachy@ensea.fr) \\ ** CReSTIC-Université de Reims Champagne Ardenne - Reims \& EPI \\ Non-A, INRIA (Mamadou.Mboup@univ-reims.fr) \\ *** LAGIS (FRE CNRS 3303) ECLille \&3 EPI Non-A, INRIA Lille \\ Ecole Centrale de Lille, BP 48, Cité Scientifique, 59651 Villeneuve \\ d'Ascq, France.(Jean-Pierre.Richard@ec-lille.fr)
}

\begin{abstract}
In this article, we propose a multidimensional numerical differentiation method from irregularly sampled data, in a noisy environment. The method extends to irregular grids our previous work and it relies on the same spirit as the algebraic numerical differentiators due to M. Mboup et al. ("Numerical differentiation with annihilators in noisy environment," Numerical Algorithm, 50, pp. 439-457, 2009). We provide a least squares interpretation of the developed estimators as well as a discrete implementation scheme. Several numerical simulations are presented to asses the performance of the proposed differentiators.
\end{abstract}

Keywords: Numerical differentiation, parameter estimation, irregular sampling.

\section{INTRODUCTION}

Numerical differentiation of noisy data is a recurrent and longstanding problem. It has attracted a lot of attention in the monovariable case (Anderssen and Bloomfield [1974], Cullum [1971], Diop et al. [2000], Ibrir and Diop [2004], Fliess and Join [2009], Levant [2003], Mboup et al. [2007, 2009], Rangarajana and Purushothaman [2009]) and multivariable case (Bachalany et al. [2010], Riachy et al. [2008, 2010]). This communication is devoted to numerical differentiation from irregularly collected data in the presence of noise.

Regular sampling has long been perceived as the most adequate way of collecting numerical data. Moreover, this perception is further strengthened by the nice sampling theorem of Nyquist-Shannon which has allowed the wide development of information theory. Nonetheless, in many practical situations, it may happen that the data are collected on irregular grids. It is, in particular, the case for seismic measurements Herrmann and Hennenfent [2008] where the data are often irregular and sparse. Another example arises from the coverage control with distributed mobile sensing robots Schwager et al. [2009] where the goal is to bring a network of robots to spread out over a region to explore and collect information about a physical quantity while aggregating in areas where the quantity of interest is at a high level.

In addition to these situations, which we may qualify as accidental or stemming from physical constrains, the preeminence itself of the regular sampling is now questioned by the emerging field of compressed sensing or compressed sampling Donoho [2006]. This new paradigm, which fully

* This work was supported in part by the PEPS project SURFER of the CNRS and by the SyMeR project of ENSEA. exploits the unnecessary character of the Nyquist criterion for perfect reconstruction, provides a renewed interest for developing numerical method based on irregularly sampled data.

This paper extends the multivariable differentiation techniques developed in Bachalany et al. [2010], Riachy et al. $[2008,2010]$ to irregular grids. While there exists few studies on numerical differentiation on irregular grids, interpolation of unorganized data (for 3-D shape reconstruction) has been the subject of a lot of studies (see Hoppe et al. [1992] for example). So, numerical differentiation could be approached by first resorting to such an interpolation method and then differentiating the resulting interpolant function. However, we consider a more direct method based on pointwise derivative estimation. As in Bachalany et al. [2010], Riachy et al. [2008, 2010], the method is inspired from the recent algebraic differentiators introduced by Mboup et al. [2009], within the framework of the algebraic estimation theory initiated in Fliess and Sira-Ramirez [2003].

For simplicity's sake, the differentiation method is developed for two dimensional signals. The extension to higher dimensions is straightforward. The departure point is the classical assumption that on a small convex domain $\mathcal{D} \subset \mathbb{R}^{2}$, the signal can be approximated by a convergent Taylor expansion. The desired derivative is expressed in terms of integrals of the measured signal. We point out that the synthesis of the estimators reduces considerably if the domain $\mathcal{D}$ is rectangular.

The communication starts with a brief review of the numerical differentiation method on regular grid of Bachalany et al. [2010], Riachy et al. [2010]. In section 3 we generalize the method to irregular grids and provide point- 
wise estimators. We show that the developed estimators can be written in a truncated Jacobi polynomial basis with a special weighing function leading to a link with least squares. In section 4 numerical simulations point out the validity of our derivative estimators. In the first (resp. second) simulation, we estimate the first (resp. second) order partial derivative of an academic noisy signal. In the third simulation, we consider the estimation of a physical parameter in a first order partial differential equation.

\section{NUMERICAL DIFFERENTIATION ON A REGULAR GRID: A BRIEF OVERVIEW}

Let us consider with Bachalany et al. [2010], Riachy et al. [2010] a signal $I(x, y)$ measured through an additive noise $\varpi(x, y)$. The variables $x$ and $y$ are independent. The measured signal $J(x, y)$ reads:

$$
J(x, y)=I(x, y)+\varpi(x, y) .
$$

The structured part $I(x, y)$ is assumed to admit a convergent Taylor series expansion:

$$
I(x, y)=\sum_{i, j} I_{x^{i} y^{j}}(0,0) \frac{x^{i} y^{j}}{i ! j !},
$$

where $I_{x^{i} y^{j}}(0,0)$ denote $\frac{\partial^{i+j}}{\partial x^{i} \partial y^{j}} I(0,0)$. Our goal is to estimate $I_{x^{n} y^{m}}(0,0)$. A truncation of $(2)$ to the $N^{\text {th }}$ order $(N \geq n)$ in $x$ and the $M^{t h}$ order $(M \geq m)$ in $y$ gives:

$$
\mathbb{I}_{N, M}(x, y)=\sum_{i=0}^{N} \sum_{j=0}^{M} I_{x^{i} y^{j}}(0,0) \frac{x^{i} y^{j}}{i ! j !} .
$$

Next, apply (for $x \geq 0$ and $y \geq 0$ ) two Laplace transforms to $(3)$ :

$$
\hat{\mathbb{I}}_{N, M}(s, p)=\sum_{i=0}^{N} \sum_{j=0}^{M} \frac{\left.I_{x^{i} y^{j}}(0,0)\right)}{s_{1}^{i+1} s_{2}^{j+1}},
$$

where $s_{1}$ (resp. $s_{2}$ ) is the operational analogue of $x$ (resp. $y)$. Let us extract the desired $(n, m)^{t h}$ order derivative $I_{x^{n} y^{m}}(0,0)$ from $(4)$ by applying the differential operator $^{1}$ :

where

$$
\Pi_{\kappa_{1}, \mu_{1}, \kappa_{2}, \mu_{2}}^{N, n, M, m}=\Xi_{\kappa_{1}, \mu_{1}}^{N, n} \Lambda_{\kappa_{2}, \mu_{2}}^{M, m}
$$

$$
\Xi_{\kappa_{1}, \mu_{1}}^{N, n}=\frac{1}{s_{1}^{N+\mu_{1}+1}} \frac{d^{n+\kappa_{1}}}{d s_{1}^{n+\kappa_{1}}} \frac{1}{s_{1}} \frac{d^{N-n}}{d s_{1}^{N-n}} s_{1}^{N+1},
$$

and

$$
\Lambda_{\kappa_{2}, \mu_{2}}^{M, m}=\frac{1}{s_{2}^{M+\mu_{2}+1}} \frac{d^{m+\kappa_{2}}}{d s_{2}^{m+\kappa_{2}}} \frac{1}{s_{2}} \frac{d^{M-m}}{d s_{2}^{M-m}} s_{2}^{M+1} .
$$

Finally by applying the inverse Laplace transform we obtain a family of estimators of the derivative $I_{x^{n}} y^{m}(0,0)$ parameterized by the integers $\kappa_{1}, \mu_{1}, \kappa_{2}$ and $\mu_{2}$.The general form of the estimators is given by:

$$
I_{x^{n} y^{m}}(0,0)=\int_{0}^{X} \int_{0}^{Y} E(X, Y, x, y) J(x, y) d x d y,
$$

where $E$ is a polynomial in $x$ and $y$ while $X$ and $Y$ are any strictly positive constants and $[0, X] \times[0, Y]$ is a rectangular domain on which the integrals are evaluated.

In Riachy et al. [2010], Bachalany et al. [2010] a detailed study of the structural properties of the estimators (5) was

\footnotetext{
1 Lack of space prenvents us from reproducing detailed computations.
}

conducted. A least squares interpretation was provided as well as a discrete implementation scheme. Since regularly spaced data was assumed therein, the implementation scheme reduced to FIR filtering. Unfortunately, this is no longer possible on irregular grids, though, we will see that the numerical complexity is still low with our method.

The work in Riachy et al. [2010], Bachalany et al. [2010] consisted of a multivariable extension of Mboup et al. [2009] inspired from algebraic techniques initiated in Fliess and Sira-Ramirez [2003].

\section{NUMERICAL DIFFERENTIATION ON IRREGULAR GRIDS}

We consider the estimation of the $(n, m)^{t h}$ order partial derivative of a smooth signal $I(x, y)$ defined on a convex domain $\mathbb{D} \subset \mathbb{R}^{2}$, from some noisy observation $J(x, y)$, as in (1). Without loss of generality, we will devise the derivative estimators around $(0,0)$. Derivative estimation at points $(\bar{x}, \bar{y})$ different from $(0,0)$ is realized by translation. To begin, we replace the rectangular domain of section 2 by any convex domain. Let then $\mathcal{D} \subset \mathbb{D}$ be any such domain around $(0,0)$. Setting

$$
a=\inf _{(x, y) \in \mathcal{D}}\{x\} ; \quad b=\sup _{(x, y) \in \mathcal{D}}\{x\}
$$

then there exists two continuous functions $f_{1}$ and $f_{2}$ : $[a, b] \rightarrow \mathbb{R}$ such that

$$
\forall(x, y) \in \mathcal{D}, a \leqslant x \leqslant b, \quad \text { and } f_{1}(x) \leqslant y \leqslant f_{2}(x) .
$$

Of course, one can replace $x$ by $y$ and obtain similar description of $\mathcal{D}$. Let then denote by $c, d$ the infimum and supremum of $\mathcal{D}$ relative to the $y$ axis and by $g_{1}(y)$ and $g_{2}(y)$ the analogue of $f_{1}$ and $f_{2}$ respectively.

\subsection{Derivative estimators}

In order to derive our estimators we ignore the noise for a moment and rewrite (3) for $N=n, M=m$ with the simplified notation $\mathbb{I}(x, y)$ for $\mathbb{I}_{N, M}(x, y)$ :

$$
\mathbb{I}(x, y)=\sum_{i=0}^{n} \sum_{j=0}^{m} I_{x^{i} y^{j}}(\overline{0}) \frac{x^{i} y^{j}}{i ! j !}
$$

where $\overline{0}$ stands for $(0,0)$. Differentiate (7) $n$ times with respect to $x$ and $m$ times with respect to $y$ :

$$
\frac{\partial^{n+m}}{\partial x^{n} \partial y^{m}} \mathbb{I}(x, y)=I_{x^{n} y^{m}}(\overline{0}) \text {. }
$$

Let $\alpha_{1} \geq n, \beta_{1} \geq n, \alpha_{2} \geq 2 m$ and $\beta_{2} \geq 2 m$ be any four integers. Then, by multiplying equation (8) by

$G_{\beta_{1}, \beta_{2}}^{\alpha_{1}, \alpha_{2}}(x, y)=(b-x)^{\alpha_{1}}(a-x)^{\beta_{1}}\left(f_{2}(x)-y\right)^{\alpha_{2}}\left(f_{1}(x)-y\right)^{\beta_{2}}$ and integrating over $\mathcal{D}$, we isolate the desired partial derivative:

$$
I_{x^{n} y^{m}}(\overline{0})=\frac{\int_{a}^{b} \int_{f_{1}(x)}^{f_{2}(x)} G_{\beta 1, \beta_{2}}^{\alpha_{1}, \alpha_{2}}(x, y) \frac{\partial^{n+m} \mathbb{I}(x, y)}{\partial x^{n} \partial y^{m}} d x d y}{\int_{a}^{b} \int_{f_{1}(x)}^{f_{2}(x)} G_{\beta 1, \beta_{2}}^{\alpha_{1}, \alpha_{2}}(x, y) d x d y} .
$$

An estimator $\widetilde{I}_{x^{n} y^{m}}$ of $I_{x^{n} y^{m}}(\overline{0})$ is therefore obtained by replacing the unavailable truncated Taylor series $\mathbb{I}(x, y)$ by the actual noisy observation $J(x, y)$ : 


$$
\widetilde{I}_{x^{n} y^{m}}(\overline{0} ; \bullet)=\frac{\int_{a}^{b} \int_{f_{1}(x)}^{f_{2}(x)} G_{\beta 1, \beta_{2}}^{\alpha_{1}, \alpha_{2}}(x, y) \frac{\partial^{n+m} J(x, y)}{\partial x^{n} \partial y^{m}} d x d y}{\int_{a}^{b} \int_{f_{1}(x)}^{f_{2}(x)} G_{\beta 1, \beta_{2}}^{\alpha_{1}, \alpha_{2}}(x, y) d x d y} .
$$

Formula (10) provides a pointwise derivative estimator at $(0,0)$. Note that this estimator varies with the choice of the parameters $\alpha_{i}$ and $\beta_{i}, i=1,2$. This dependance is quoted by the notation above where $(\bullet)$ refers to $\left(\alpha_{1}, \alpha_{2}, \beta_{1}, \beta_{2}\right)$. As mentioned earlier, a pointwise derivative estimator at $(\bar{x}, \bar{y}) \neq(0,0)$ is accomplished by translation, that is by replacing the signal $J(x, y)$ in (10) by its translated version $J(x+\bar{x}, y+\bar{y})$. When there is no risk of confusion, the superscripts and the subscripts will be omitted from $G_{\beta_{1}, \beta_{2}}^{\alpha_{1}, \alpha_{2}}(x, y)$ and write $G(x, y)$ for short. Next, we show that (10) corresponds to an orthogonal projection in a properly chosen orthogonal polynomial basis function.

\subsection{Least squares interpretation}

Inspired by Mboup [2009], let us consider the Jacobi polynomials $\left\{\mathcal{P}_{q, p}^{\left(\alpha_{1}, \alpha_{2}, \beta_{1}, \beta_{2}\right)}(x, y)\right\}, p, q=0,1, \ldots$, orthogonal on the interval $[a, b] \times\left[f_{1}(x), f_{2}(x)\right]$ with respect to the weighing function $G(x, y)$. They can be derived using the following (Rodriguez) differential equation adapted from Abramowitz and Stegun [1965]:

$$
G(x, y) \mathcal{P}_{q, p}^{\left(\alpha_{1}, \alpha_{2}, \beta_{1}, \beta_{2}\right)}=\frac{(-1)^{q+p}}{q ! p !} \frac{\partial^{q} P(x)}{\partial x^{q}} \frac{\partial^{p} Q(x, y)}{\partial y^{p}}
$$

where $P(x)=(b-x)^{\alpha_{1}}(a-x)^{\alpha_{2}}$ and $Q(x, y)=\left(f_{1}(x)-\right.$ $y)^{\beta_{1}}\left(f_{2}(x)-y\right)^{\beta_{2}}$. Note that $\mathcal{P}_{0,0}=1$. Let the $(q, p)^{t h}$ order least square approximation of a function $\mathcal{F}(x, y)$ be defined by:

$$
\mathcal{F}_{L S,(q, p)}(\xi, \zeta)=\sum_{i=0}^{q} \sum_{j=0}^{p} \frac{\left\langle\mathcal{P}_{i, j}^{(\bullet)}(x, y), \mathcal{F}(x, y)\right\rangle}{\left\|\mathcal{P}_{i, j}^{(\bullet)}\right\|^{2}} \mathcal{P}_{i, j}^{(\bullet)}(\xi, \zeta),
$$

where $(\bullet)$ still stands for $\left(\alpha_{1}, \alpha_{2}, \beta_{1}, \beta_{2}\right)$. We have:

Proposition 3.1. The derivative estimator (10) is given by

$$
\widetilde{I}_{x^{n} y^{m}}(\overline{0} ; \bullet)=\mathcal{F}_{L S,(1,1)}\left(\xi_{0}, \zeta_{0}\right)+\Delta_{\varpi, n, m}(0,0)
$$

for $\mathcal{F}(x, y)=\frac{\partial^{n+m} I(x, y)}{\partial x^{n} \partial y^{m}}$ and where $\Delta_{\varpi, n, m}(0,0)$ represents both the noise and the truncation error contributions and where $\left(\xi_{0}, \zeta_{0}\right)$ is the zero of $\mathcal{P}_{1,1}^{(\bullet)}$.

Proof. The proof follows the same lines as in Bachalany et al. [2010], Riachy et al. [2010]

This proposition shows that the estimator (10) corresponds to the derivative estimation at a delayed point given by $\left(\xi_{0}, \zeta_{0}\right)$, although it is derived from a Taylor expansion around zero (see Mboup et al. [2009] for more details).

Remark 1. When $\alpha_{1}=\beta_{1}$ and $\alpha_{2}=\beta_{2}$, we have $\xi_{0}=\frac{a+b}{2}$ and $\zeta_{0}=\frac{f_{1}\left(\frac{a+b}{2}\right)+f_{2}\left(\frac{a+b}{2}\right)}{2}$. Therefore, it is preferable to have a domain $\mathcal{D}$ symmetric with respect to $(0,0)$ in order to reduce the delay or even cancel it when $a=-b$ and $f_{1}(x)=-f_{2}(x)$.

\subsection{Higher order approximations and interpolation}

In the previous subsection we showed that the pointwise derivative estimator is equivalent to a delayed first order least squares approximation, with a fixed delay. Here, we show that the delay can be set to any point $(\xi, \zeta) \in \mathcal{D}$ by a judicious linear combination of the previous estimators. We also show that a higher order approximation can be achieved by a proper selection of the delay.

For this, let us consider the following linear combination:

$$
\begin{aligned}
\mathcal{H} & =\lambda_{1} \widetilde{I}\left(\overline{0} ; \alpha_{1}+1, \beta_{1}, \alpha_{2}, \beta_{2}\right)+\lambda_{2} \widetilde{I}\left(\overline{0} ; \alpha_{1}, \beta_{1}+1, \alpha_{2}, \beta_{2}\right) \\
& +\lambda_{3} \widetilde{I}\left(\overline{0} ; \alpha_{1}, \beta_{1}, \alpha_{2}+1, \beta_{2}\right)+\lambda_{4} \widetilde{I}\left(\overline{0} ; \alpha_{1}, \beta_{1}, \alpha_{2}, \beta_{2}+1\right) \\
& +\lambda_{5} \widetilde{I}\left(\overline{0} ; \alpha_{1}, \beta_{1}, \alpha_{2}, \beta_{2}\right) ; \lambda_{1}, \cdots, \lambda_{5} \in \mathbb{R} .
\end{aligned}
$$

Let $\mathcal{G}_{\beta 1, \beta_{2}}^{\alpha_{1}, \alpha_{2}}=\int_{a}^{b} \int_{f_{1}(x)}^{f_{2}(x)} G(x, y) d x d y$ and set $\lambda_{1}^{\prime}=\frac{\lambda_{1}}{\mathcal{G}_{\beta 1, \beta_{2}}^{\alpha_{1}+\alpha_{2}}}$, $\lambda_{2}^{\prime}=\frac{\lambda_{2}}{\mathcal{G}_{\beta 1+1, \beta_{2}}^{\alpha_{1}, \alpha_{2}}}, \lambda_{3}^{\prime}=\frac{\lambda_{3}}{\mathcal{G}_{\beta 1, \beta_{2}}^{\alpha_{1}, \alpha_{2}+1}}, \lambda_{4}^{\prime}=\frac{\lambda_{4}}{\mathcal{G}_{\beta 1, \beta_{2}+1}^{\alpha_{1}, \alpha_{2}}}, \lambda_{5}^{\prime}=\frac{\lambda_{5}}{\mathcal{G}_{\beta 1, \beta_{2}}^{\alpha_{1}, \alpha_{2}}}$.

Then, equation (13) reads as:

$$
\mathcal{H}=\int_{a}^{b} \int_{f_{1}(x)}^{f_{2}(x)} H(x, y) G(x, y) \frac{\partial^{n+m} J(x, y)}{\partial x^{n} \partial y^{m}} d x d y
$$

with $H(x, y)$ given by:

$$
\begin{aligned}
H(x, y) & =\lambda_{1}^{\prime}(b-x)+\lambda_{2}^{\prime}(a-x)+\lambda_{3}^{\prime}\left(f_{2}(x)-y\right) \\
& +\lambda_{4}^{\prime}\left(f_{1}(x)-y\right)+\lambda_{5}^{\prime} .
\end{aligned}
$$

Proposition 3.2. Let $(\xi, \zeta)$ be any point in $\mathcal{D}$. Setting $\lambda_{1}^{\prime}=$ $\frac{\left(\beta_{1}+1\right) \mathcal{P}_{1,0}^{(\bullet)}(\xi, \zeta)}{\left\|\mathcal{P}_{1,0}^{(\bullet)}\right\|^{2}}, \lambda_{2}^{\prime}=\frac{\left(\alpha_{1}+1\right) \mathcal{P}_{1,0}^{(\bullet)}(\xi, \zeta)}{\left\|\mathcal{P}_{1,0}^{(\bullet)}\right\|^{2}}, \lambda_{3}^{\prime}=\frac{\left(\beta_{2}+1\right) \mathcal{P}_{0,1}^{(\bullet)}(\xi, \zeta)}{\left\|\mathcal{P}_{0,1}^{(\bullet \bullet}\right\|^{2}}$, $\lambda_{4}^{\prime}=\frac{\left(\alpha_{2}+1\right) \mathcal{P}_{0,1}^{(\bullet)}(\xi, \zeta)}{\left\|\mathcal{P}_{0,1}^{(\bullet)}\right\|^{2}}, \lambda_{5}^{\prime}=\frac{1}{\left\|\mathcal{P}_{0,0}^{(\bullet)}\right\|^{2}}$, we have:

$$
\mathcal{H}=\mathcal{F}_{L S,(1,1)}(\xi, \zeta)+\Delta_{\varpi, n, m}(\xi, \zeta)
$$

for $\mathcal{F}(x, y)=\frac{\partial^{n+m} I(x, y)}{\partial x^{n} \partial y^{m}}$. The noise and truncation error contributions are represented by $\Delta_{\varpi, n, m}(\xi, \zeta)$.

As a direct consequence, if we choose $(\xi, \zeta)$ in (14) to correspond to a zero of the second order Jacobi polynomial we get a second order least squares approximation.

Recall that traditionally, numerical differentiation is approached via a least square interpolation followed by a differentiation of the resulting interpolant function. It is important to point out that our approach directly provides a least square approximation of the derivative. Moreover, the derivatives are approximated locally and pointwise.

\subsection{Implementable estimators}

Examining the estimator (10) we can see that partial derivatives of the noisy signal appear under the integrals. To get rid of the derivatives integrations by parts are performed. Note that $G(x, y)$ was intentionally chosen such that it vanishes on $a, b, f_{1}$ and $f_{2}$ in order to get rid of the boundary conditions. Next, we put the first and second order derivatives into implementable forms.

Estimator for $I_{x}(\overline{0})$ and $I_{y}(\overline{0})$ : Let us consider the estimator for $I_{y}(\overline{0})$, corresponding to $n=0, m=1$, with 
the simple choice $\alpha_{1}=\beta_{1}=0$ and $\alpha_{2}=\beta_{2}=1$. By integrating by parts the numerator of (10), we obtain:

$$
\widetilde{I}_{y}(\overline{0} ; \bullet)=\frac{\int_{a}^{b} \int_{f_{1}(x)}^{f_{2}(x)}\left(f_{1}+f_{2}-2 y\right) J(x, y) d x d y}{\int_{a}^{b} \int_{f_{1}(x)}^{f_{2}(x)}\left(f_{1}-y\right)\left(f_{2}-y\right) d x d y} .
$$

Similarly an estimator for $I_{x}(\overline{0})$ is obtained by:

$$
\widetilde{I}_{x}(\overline{0} ; \bullet)=\frac{\int_{c}^{d} \int_{g_{1}(y)}^{g_{2}(y)}\left(g_{1}+g_{2}-2 x\right) J(x, y) d y d x}{\int_{c}^{d} \int_{g_{1}(y)}^{g_{2}(y)}\left(g_{1}-x\right)\left(g_{2}-x\right) d y d x} .
$$

Estimator for $I_{x^{2}}(\overline{0})$ and $I_{y^{2}}(\overline{0})$ : Taking $\alpha_{1}=\beta_{1}=0$, $\alpha_{2}=\beta_{2}=2$, in (10) and integrating by parts twice we obtain, for $n=0, m=2$ :

$$
\widetilde{I}_{y^{2}}(\overline{0} ; \bullet)=\frac{\int_{a}^{b} \int_{f_{1}(x)}^{f_{2}(x)} \mathcal{G}_{1}(x, y) J(x, y) d x d y}{\int_{a}^{b} \int_{f_{1}(x)}^{f_{2}(x)}\left(f_{1}-y\right)^{2}\left(f_{2}-y\right)^{2} d x d y},
$$

where $\mathcal{G}_{1}(x, y)=2\left(f_{1}+f_{2}-2 y\right)^{2}+4\left(f_{1}-y\right)\left(f_{2}-y\right)$. An estimator for $I_{x^{2}}(\overline{0})$ is deduced similarly.

Estimator for $I_{x y}(\overline{0})$ : Let us first introduce the new variables

$$
u=x, \quad v=\frac{y-f_{1}(u)}{f_{2}(u)-f_{1}(u)},
$$

and define $\mathcal{J}$ by $J(x, y)=\mathcal{J}(u(x, y), v(x, y))$. The partial derivative $\frac{\partial^{2} J(x, y)}{\partial x \partial y}$ may be expressed as:

$$
\begin{aligned}
\frac{\partial^{2} J(x, y)}{\partial x \partial y} & =\frac{\partial^{2} \mathcal{J}(u, v)}{\partial u \partial v} \frac{1}{f_{2}(u)-f_{1}(u)} \\
& -\frac{\partial^{2} \mathcal{J}(u, v)}{\partial v^{2}} \frac{f_{1}^{\prime}(u)+v\left(f_{2}^{\prime}(u)-f_{1}^{\prime}(u)\right)}{\left(f_{2}(u)-f_{1}(u)\right)^{2}} \\
& -\frac{\partial \mathcal{J}(u, v)}{\partial v} \frac{f_{2}^{\prime}(u)-f_{1}^{\prime}(u)}{\left(f_{2}(u)-f_{1}(u)\right)^{2}}
\end{aligned}
$$

where $f_{1}^{\prime}(u)=\frac{d f_{1}(u)}{d u}$ and $f_{2}^{\prime}(u)=\frac{d f_{2}(u)}{d u}$. Let us also define $\mathcal{K}(u, v)=(a-u)(b-u) v^{2}(v-1)^{2}\left(f_{2}(u)-f_{1}(u)\right)$. With these settings, the numerator of (10) with $n=m=1$ and using $\alpha_{1}=\beta_{1}=1$ and $\alpha_{2}=\beta_{2}=2$ becomes:

$$
\begin{aligned}
\mathcal{N} & =\int_{a}^{b} \int_{0}^{1} \mathcal{K}(u, v)\left(f_{2}-f_{1}\right) \frac{\partial^{2} \mathcal{J}(u, v)}{\partial v \partial u} d u d v \\
& -\int_{a}^{b} \int_{0}^{1} \mathcal{K}(u, v)\left(f_{1}^{\prime}+v\left(f_{2}^{\prime}-f_{1}^{\prime}\right)\right) \frac{\partial^{2} \mathcal{J}(u, v)}{\partial v^{2}} d u d v \\
& -\int_{a}^{b} \int_{0}^{1} \mathcal{K}(u, v)\left(f_{2}^{\prime}-f_{1}^{\prime}\right) \frac{\partial \mathcal{J}(u, v)}{\partial v} d u d v
\end{aligned}
$$

To the first double integral of (20), we apply the double integration by parts formula from Ghorpade and Limaye [2010] that we recall hereafter for completeness.

Proposition 3.3. Double integration by parts Ghorpade and Limaye [2010] Let $\mathfrak{R}:=[\mathfrak{a}, \mathfrak{b}] \times[\mathfrak{c}, \mathfrak{d}]$ and let $\mathfrak{h}(x, y), \mathfrak{g}(x, y), \mathfrak{k}(x, y): \mathfrak{R} \rightarrow \mathbb{R}$ be integrable functions satisfying the following:

- The functions $\mathfrak{h}$ and $\mathfrak{k}$ are continuous in the first variable.
- $\mathfrak{h}_{x}, \mathfrak{h}_{y}, \mathfrak{h}_{x y}, \mathfrak{k}_{x}$ and $\mathfrak{k}_{y}$ exist and are integrable on $\mathfrak{R}$.

- The functions $\mathfrak{h}_{x}$ and $\mathfrak{k}_{x}$ are continuous in the second variable.

- $\mathfrak{k}_{x y}$ exists and $\mathfrak{k}_{x y}=\mathfrak{g}$ on $\mathfrak{R}$.

Then

$$
\iint_{\mathfrak{R}} \mathfrak{h} \mathfrak{g}=\Delta_{(\mathfrak{b}, \mathfrak{d})}^{(\mathfrak{a}, \mathfrak{c})}(\mathfrak{h} \mathfrak{k})-\iint_{\mathfrak{R}}\left(\mathfrak{h}_{x} \mathfrak{k}_{y}+\mathfrak{h}_{y} \mathfrak{k}_{x}+\mathfrak{h}_{x y} \mathfrak{k}\right)
$$

where

$\Delta_{(\mathfrak{a}, \mathfrak{c})}^{(\mathfrak{b}, \mathfrak{d})}(\mathfrak{h} \mathfrak{k}):=(\mathfrak{h} \mathfrak{k})(\mathfrak{b}, \mathfrak{d})-(\mathfrak{h} \mathfrak{k})(\mathfrak{b}, \mathfrak{c})-(\mathfrak{h} \mathfrak{k})(\mathfrak{a}, \mathfrak{d})+(\mathfrak{h} \mathfrak{k})(\mathfrak{a}, \mathfrak{c})$.

Applying (21) to the first integral of equation (20) yields:

$$
\begin{aligned}
& \mathcal{N}=-\int_{a}^{b} \int_{0}^{1} \mathcal{K}_{1}\left(f_{2}-f_{1}\right)(2 u-a-b) \mathcal{J}(u, v) d u d v \\
& -\int_{a}^{b} \int_{0}^{1} \mathcal{K}_{2}\left(12 v^{2}-12 v+2\right)\left[f_{1}^{\prime}+v\left(f_{2}^{\prime}-f_{1}^{\prime}\right)\right] \mathcal{J}(u, v) d u d v \\
& -6 \int_{a}^{b} \int_{0}^{1} \mathcal{K}_{1} \mathcal{K}_{2}\left(f_{2}^{\prime}-f_{1}^{\prime}\right) \mathcal{J}(u, v) d u d v
\end{aligned}
$$

with $\mathcal{K}_{1}=2 v(v-1)(2 v-1)$ and $\mathcal{K}_{2}=(a-u)(b-u)\left(f_{2}-f_{1}\right)$. Finally, the estimator reads as:

$$
\widetilde{I}_{x y}(\overline{0} ; \bullet)=\frac{\mathcal{N}}{\int_{a}^{b} \int_{f_{1}(x)}^{f_{2}(x)} \mathcal{G}(x, y) d x d y} .
$$

Remark that in order to estimate $\widetilde{I}_{x y}(\overline{0}, \bullet), f_{1}(x)$ and $f_{2}(x)$ should be at least differentiable.

\subsection{Rectangular domain}

In many situations, the integration domain $\mathcal{D}$ can be rectangular. The derivative estimator formulae become considerably simpler. In fact the pointwise estimator formula (10) evaluated on the rectangular domain $\mathcal{D}=[a, b] \times[c, d]$ reads as:

$$
\widetilde{I}_{x^{n} y^{m}}(\overline{0}, \bullet)=\frac{\int_{a}^{b} \int_{c}^{d} \frac{\partial^{n+m} G(x, y)}{\partial x^{n} \partial y^{m}} J(x, y) d x d y}{\int_{a}^{b} \int_{f_{1}(x)}^{f_{2}(x)} G(x, y) d x d y} .
$$

Note, for example, that for $\alpha_{1}=\beta_{1}=\alpha_{2}=\beta_{2}=1$ and $n=m=1$, the following estimator:

$$
\widetilde{I}_{x, y}(\overline{0} ; \bullet)=\frac{\int_{a}^{b} \int_{c}^{d}(a+b-2 x)(c+d-2 y) J(x, y) d x d y}{\int_{a}^{b} \int_{c}^{d}(a+b-2 x)(c+d-2 y) d x d y}
$$

can be used in the place of (22).

\subsection{Implementation}

The integrals involved in the estimators are discretized and numerically evaluated. For the pointwise derivative estimation at a given point in $\mathbb{D}$, all measurements points within a given distance are considered. The convex hull of these points defines the convex domain $\mathcal{D} \subset \mathbb{D}$. Then a Delaunay triangulation algorithm is applied on the points of interests. At each triangle the trapezoidal rule is applied to approximate the integral. More precisely the Delaunay triangulation consists of, say, $L$ triangles $\left\{T_{i}\right\}_{i=1}^{L}$ such that 
$\mathcal{D}=\cup_{i=1}^{L} T_{i}$ and $T_{i} \cap T_{j}=\emptyset \forall i \neq j$. The estimator in equation (15), for example, is computed as:

$$
\widetilde{I}_{y}(\overline{0} ; \bullet)=\frac{\sum_{i=1}^{L} \int_{T_{i}}\left(f_{1}+f_{2}-2 y\right) J(x, y) d x d y}{\sum_{i=1}^{L} \int_{T_{i}}\left(f_{1}-y\right)\left(f_{2}-y\right) d x d y} .
$$

For each integral in (23) the integrand is evaluated at the vertices of the corresponding triangle $T_{i}$, then summed up, divided by three and multiplied by the area of $T_{i}$.

\section{NUMERICAL SIMULATIONS}

In this section, we provide numerical simulation of the first and second order derivative estimators as well as a parameter identification example. An extensive numerical study will be published in a an extended future work.

\subsection{First order derivative estimation}

As a first example, we are interested in estimating the first order derivative of:

$$
\mathcal{T}(x, y)=\cos (2(x+y)) .
$$

A slice of (24) at $y=0$ is depicted in Figure 1. The square domain $\mathbb{D}=[-2.5,2.5] \times[-2.5,2.5]$ is considered and 10000 points are generated randomly to simulate an irregular sampling. The derivative estimate is evaluated, however, on the regular grid $\mathcal{O}=[-2,-1.9, \cdots, 1.9,2]$ $\times[-2,-1.9, \cdots, 1.9,2]$. At each point $\left(x_{o}, y_{o}\right)$ of $\mathcal{O}$ equation (23) is evaluated using the convex domain: $\mathcal{D}=$ $\left\{(x, y) ;\left(x-x_{o}\right)^{2}+\left(y-y_{o}\right)^{2}<0.15\right\}$. A white noise corrupts the signal as shown in the Figure 1. The estimation of $\frac{\partial \mathcal{T}}{\partial x}(\bar{x}, 0), \bar{x} \in[-2,-1.9, \cdots, 1.9,2]$ is shown in the Figure 2 . An example of a grid used to evaluate the estimators is given in the Figure 3.

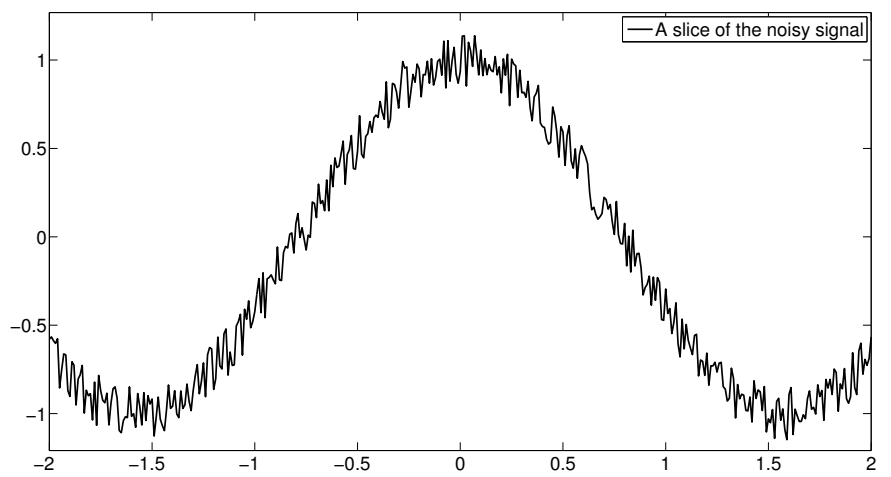

Fig. 1. A slice of the noisy signal.

\subsection{Second order derivative estimation}

We are interested here in estimating the second order derivative of (24) with an additive noise as shown in Figure 1 on $\mathbb{D}=[-2.5,2.5] \times[-2.5,2.5]$. We randomly generate 100000 points in $\mathbb{D}$. The derivative is calculated on the regular grid given by $\mathcal{O}$ above. At each point $\left(x_{o}, y_{o}\right) \in \mathcal{O}$ a discretization of equation (16) is evaluated using $\mathcal{D}=\left\{(x, y) ;\left(x-x_{o}\right)^{2}+\left(y-y_{o}\right)^{2}<0.2\right\}$. The estimation of $\frac{\partial^{2} \mathcal{T}}{\partial x^{2}}(\bar{x}, 0)$, is shown in the Figure 4 .

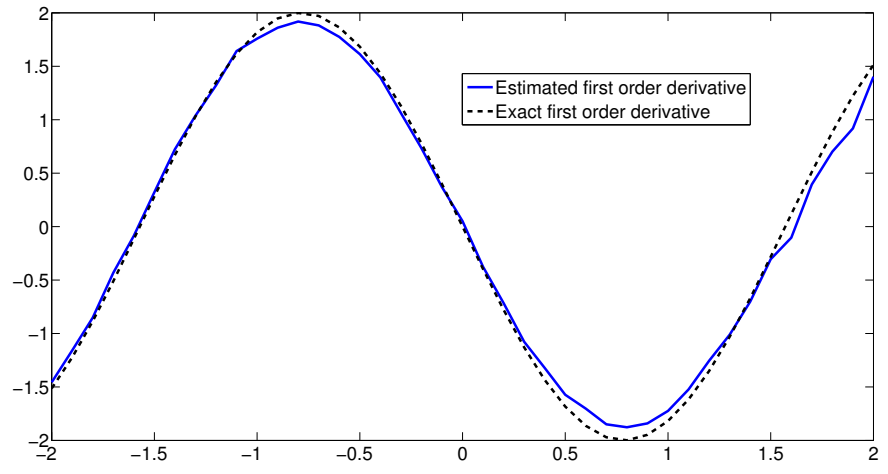

Fig. 2. Exact (dashed line) and estimated (solid line) values of $\frac{\partial \mathcal{T}}{\partial x}$.

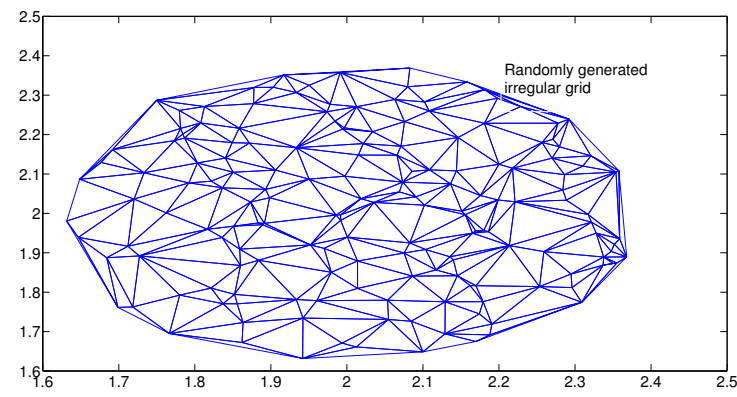

Fig. 3. A randomly generated grid used for the estimation of $\mathcal{T}_{x}$ with $(x-2)^{2}+(y-2)^{2}<0.15$.

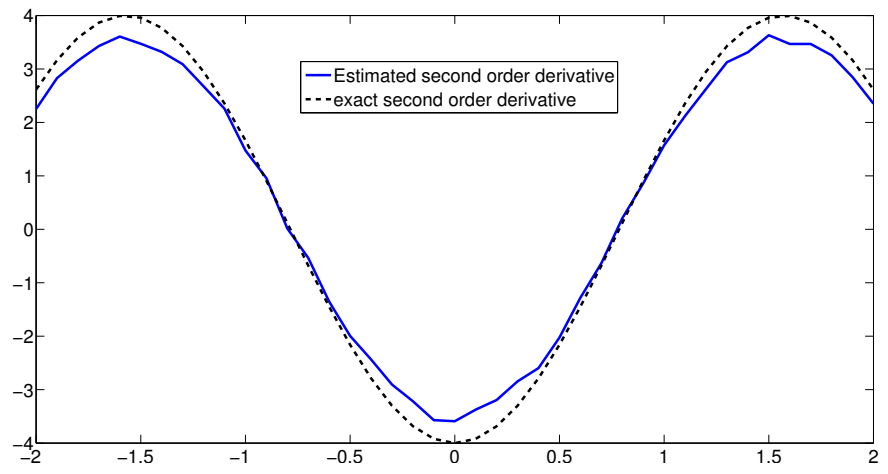

Fig. 4. Exact (dashed line) and estimated (solid line) values of $\frac{\partial^{2} \mathcal{T}}{\partial x^{2}}$.

\subsection{Parameter estimation example}

In this section, we consider an example of a fluid mechanics problem. We are interested in estimating the thermal conductivity $\kappa(x, y)$ of a fluid, from the measurements of the heat flow rate $q(x, y)$ and the temperature $\mathcal{R}$.

The three quantities are related by the partial differential equation $q(x, y)=\kappa(x, y)\left(\frac{\partial \mathcal{R}(x, y)}{\partial x}+\frac{\partial \mathcal{R}(x, y)}{\partial y}\right)$. We assume that some (inevitably noisy) measurements of $q$ and $\mathcal{R}$ are available on an irregular grid. The coefficient $\kappa(x, y)$ indeed may vary because the material can be heterogeneous. However, we suppose that $\kappa(x, y)$ is slowly varying such that we can consider it constant on the small domain $\mathcal{D}$. An estimation of $\kappa$ at a particular point $(\bar{x}, \bar{y})$ is given by :

$$
\kappa(\bar{x}, \bar{y})=\frac{\tilde{q}_{0}(\bar{x}, \bar{y})}{\widetilde{\mathcal{R}}_{x}(\bar{x}, \bar{y})+\widetilde{\mathcal{R}}_{y}(\bar{x}, \bar{y})},
$$


where $\tilde{q}_{0}(\bar{x}, \bar{y})$ is some denoised version of $q$ at $(\bar{x}, \bar{y})$, $\left(\widetilde{\mathcal{R}}_{x}, \widetilde{\mathcal{R}}_{y}\right)$ is an estimated temperature gradient. Equation (25) can be evaluated provided that $\widetilde{\mathcal{R}}_{x}(\bar{x}, \bar{y})+\widetilde{\mathcal{R}}_{y}(\bar{x}, \bar{y}) \neq$ 0 . The function $\mathcal{R}$ is simulated by $\mathcal{R}(x, y)=\cos (x+y)$ while $q$ is given by $q=-2 \sin (x+y) \cos (x / 10)$. Thus the exact value of $\kappa$ is $\kappa(x)=\cos (x / 10)$. A white noise is added to the simulation. A plot of the noisy signal $q$ is shown in figure 5. The estimated thermal conductivity is

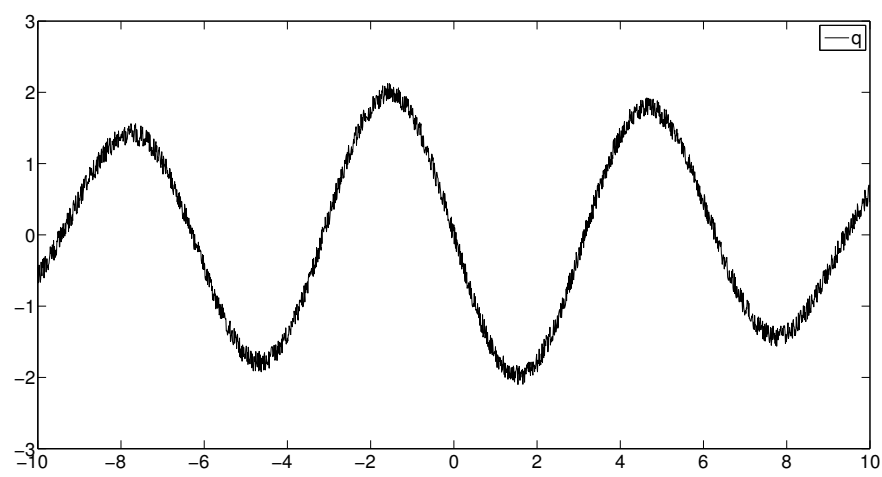

Fig. 5. Noisy plot of $q(x, 0)$.

plotted (solid line) in the figure 6 , in comparison with the exact solution (dashed line). The peak at 0 visible on figure 6 is due to the fact that both $q$ and $\mathcal{R}_{x}+\mathcal{R}_{y}$ vanishes at 0 . Except for the numerical singularity at the origin, the

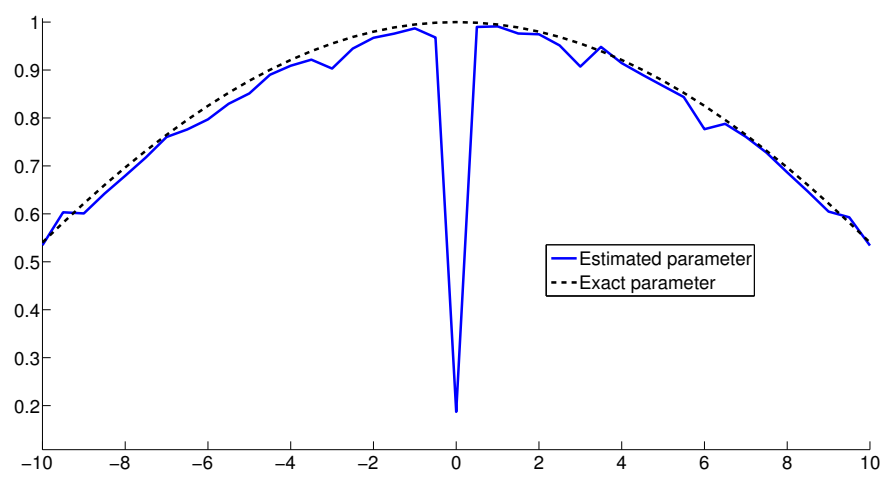

Fig. 6. Exact (dashed line) and estimated (solid line) thermal conductivity.

estimates are in good agreement with the actual values.

\section{CONCLUSION}

In this paper we developed a method for derivative estimation on irregular grid. We showed that the estimators can be expressed in a Jacobi polynomials basis yielding a link with least squares local approximation. A discrete, implementable version is proposed. Several numerical simulations attested of the performances of our estimators.

This work laid the foundations of a new numerical differentiation method on irregular grids. Several points will be treated in future works, namely:

- the choice of the domain $\mathcal{D}$ (rectangular, triangular...),

- robustness to various noises,

- application to mobile sensing robotics.

\section{REFERENCES}

M. Abramowitz and I. A. Stegun. Handbook of mathematical functions. Dover, New York, 1965.

R. S. Anderssen and P. Bloomfield. Numerical differentiation procedures for non-exact data. Numer. Math. Springer-Verlag, 22:157-182, 1974.

Y. Bachalany, S. Riachy, M. Mboup, and J.-P. Richard. Diffrentiation numérique multivariable 2 : projection orthogonale et filtrage rif. $6^{i e m e}$ Conf. Int. Francophone d'Automatique, 2010.

J. Cullum. Numerical differenciation and regularization. SIAM J. on numerical analysis, 8(2), 1971.

S. Diop, J. W. Grizzle, and F. Chaplais. On numerical differentiation algorithms for nonlinear estimation. Proc $C D C, 2000$.

D.L. Donoho. Compressed sensing. IEEE Trans. on Inf. Theory, 52(4):1289-1306, 2006.

M. Fliess and C. Join. Systematic risk analysis: first steps towards a new definition of beta. Cognitive Systems with Interactive Sensors, 2009.

M. Fliess and H. Sira-Ramirez. An algebraic framework for linear identification. ESAIM: COCV, 9:151-168, 2003.

S.R. Ghorpade and B.V. Limaye. A course in multivariable calculus and analysis. Springer, 2010.

F. J. Herrmann and G. Hennenfent. Non-parametric seismic data recovery with curvelet frames. Geophys. J. Int., 173:233-248, 2008.

H. Hoppe, T. DeRose, T. Duchamp, J. McDonald, and W. Stuetzle. Surface reconstruction from unorganized points. Int. conf. on computer graphics and interative techniques, pages 71-78, 1992.

S. Ibrir and S. Diop. A numerical procedure for filtering and efficient high-order signal differenciation. Int. J. Appl. Math. Compt. Sci., 14(201-208), 2004.

A. Levant. Higher-order sliding modes, differentiation and output-feedback control. Int. J. of Control, 76:924-941, 2003.

M. Mboup. Parameter estimation for signals described by differential equations. Applicable Analysis, 88(1):29 $52,2009$.

M. Mboup, C. Join, and M. Fliess. A revised look at numerical differentiation with an application to nonlinear feedback control. $15^{\text {th }} \mathrm{Med}$. conf. on control and automation, 2007.

M. Mboup, C. Join, and M. Fliess. Numerical differentiation with annihilators in noisy environment. Numerical Algorithm, 50:439-457, 2009.

S.K. Rangarajana and S.P. Purushothaman. Lanczos generalized derivative for higher orders. The Int. J. of Robotics Research, 177(357-755), 2009.

S. Riachy, Y. Bachalany, M. Mboup, and J.-P. Richard. An algebraic method for multi-dimensional derivative estimation. $16^{\text {th }}$ Mediterranean conference on control and automation, 2008.

S. Riachy, Y. Bachalany, M. Mboup, and J.-P. Richard. Diffrenciation numrique multivariable 1 : estimateurs algébriques et structure. $6^{\text {ieme }}$ Conf. Int. francophone d'automatique, 2010.

M. Schwager, D. Rus, and J.-J. Slotine. Decentralized, adaptive coverage control for networked robots. Int. J. of Robotics Research, 28(3):357-375, 2009. 